\title{
Heart disease and the risk of allopurinol- associated severe cutaneous adverse reactions: a general population-based cohort study
}

\author{
Chio Yokose MD, Na Lu MPH, Hui Xie MS PhD, Lingyi Li MSc, Yufei Zheng MPH, Natalie McCormick PhD, \\ Sharan K. Rai MSc, J. Antonio Aviña-Zubieta MD PhD, Hyon K. Choi MD DrPH
}

Cite as: CMAJ 2019 September 30;191:E1070-7. doi: 10.1503/cmaj.190339

\begin{abstract}
BACKGROUND: Allopurinol is commonly prescribed for gout, and its clinical use may expand with ongoing trials assessing its potential cardiorenal benefits. Because heart disease has been suggested to be a risk factor for allopurinolassociated severe cutaneous adverse reactions, we sought to confirm this association in a Canadian general population cohort.
\end{abstract}

METHODS: We used population data from British Columbia, Canada, to identify all incident allopurinol users between 1997 and 2015. We examined the association between heart disease (ischemic heart disease and heart failure) and the risk of hospital admission for severe cutaneous adverse reactions, adjusting for known and purported risk factors. We also evaluated the joint effects of combined clinical and demographic risk factors.

RESULTS: Among 130325 allopurinol initiators, 109 hospital admissions occurred for allopurinol-associated severe cutaneous adverse reactions. The multivariable relative risk among those with heart disease was 1.55 (95\% confidence interval 1.01-2.37). Patients with heart disease and chronic kidney disease who were started on an allopurinol dosage of greater than $100 \mathrm{mg} / \mathrm{d}$ had an 11-fold higher risk. Allopurinol initiation at a lower dosage among patients with heart disease and chronic kidney disease resulted in a fivefold reduction in risk. Older women with heart disease from regions with large Asian populations had a 23-fold higher risk of allopurinol-associated severe cutaneous adverse reactions than younger men without heart disease from other regions.

INTERPRETATION: Heart disease is independently associated with risk of allopurinol-associated severe cutaneous adverse reactions, similar to chronic kidney disease, and low-dosage allopurinol initiation may substantially mitigate this risk. Risk factors for these rare but serious reactions should be considered when initiating allopurinol.
$\mathrm{T}$ he global burden of gout continues to grow. ${ }^{1}$ Amid concerns regarding possible cardiovascular adverse effects with febuxostat, ${ }^{2}$ the number of patients with gout prescribed allopurinol may increase. Furthermore, as clinical trials ${ }^{3}$ investigate allopurinol's purported cardiorenal benefits, ${ }^{4,5}$ use of allopurinol among those without gout may also increase. Although generally safe and well-tolerated, allopurinol has been associated with severe cutaneous adverse reactions, including Stevens-Johnson syndrome and toxic epidermal necrolysis, which are collectively referred to as allopurinol-associated severe cutaneous adverse reactions.

A Taiwanese population-based study found that heart disease was independently associated with increased risk of hospital admission for allopurinol-associated severe cutaneous adverse reactions. ${ }^{6}$ However, this finding has not been replicated in a non-Asian cohort. We sought to fill this knowledge gap by investigating the association in a Canadian general population cohort. Moreover, we investigated the potential joint impact of heart disease and other risk factors, including chronic kidney disease, ${ }^{6-8}$ higher starting allopurinol dosage ${ }^{6,8,9}$ and demographic factors. ${ }^{6,8,10,11}$

\section{Methods}

\section{Population and study design}

We conducted a cohort study using Population Data BC, an administrative database with de-identified patient-level data on hospital discharge records, ${ }^{12}$ outpatient dispensed prescriptions ${ }^{13}$ and vital statistics for nearly all of British Columbia's 4.7 million residents. ${ }^{14}$ We used hospital admission and 
prescription data, available from 1990, to identify incident allopurinol users between 1997 and 2015, excluding individuals with a history of severe cutaneous adverse reactions before allopurinol initiation.

\section{Assessment of end points}

The primary end point was incident cases of hospital admission for allopurinol-associated severe cutaneous adverse reactions, identified based on principal hospital discharge diagnosis of a relevant International Classification of Diseases, 9th Revision, Clinical Modification (ICD-9-CM) or International Classification of Diseases, 10th Revision, Clinical Modification (ICD-10-CM) code, occurring within 3 months after filling the first prescription for allopurinol, followed by discontinuation of the drug after the hospital admission. The relevant ICD-9-CM codes included dermatitis due to drugs and medicines (693.0), erythema multiforme (695.1X) (which includes Stevens-Johnson syndrome [695.13] and toxic epidermal necrolysis [695.15]), unspecified erythematous conditions (695.9) and other specified erythematous conditions (695.89), and are consistent with those used in other claims database studies. ${ }^{6,8}$ Two expert dermatologists previously found that this method had a positive predictive value of $100 \%$ after manual chart review of 33 patients identified through a national database. ${ }^{6}$ We used a 3-month window between the first prescription for allopurinol and hospital admission for allopurinol-associated severe cutaneous adverse reactions because most of these reactions develop within 3 months of drug initiation. ${ }^{6,8,15}$ Deaths from allopurinolassociated severe cutaneous adverse reactions were defined as deaths from any cause within 60 days of hospital admission with a diagnosis of allopurinol-associated severe cutaneous adverse reaction. ${ }^{6,8}$

\section{Assessment of exposure and covariates}

The exposure of interest was heart disease (defined as ischemic heart disease and heart failure, ICD-9-CM codes 410-414 and 402, 404,428 , respectively) before allopurinol initiation. Covariates of interest consisted of known or purported risk factors, including age, sex, region (as surrogate for high-risk Asian race), presence of chronic kidney disease (ICD-9-CM codes 580-586), diabetes (ICD-9-CM code 250) and gout (ICD-9-CM code 274), use of diuretics (loop and thiazide) within 1 year before allopurinol initiation, and initial allopurinol dosage greater than $100 \mathrm{mg} / \mathrm{d}$. Age was analyzed as both a continuous variable and a categorical variable ( $<60 \mathrm{yr}, 60-70 \mathrm{yr}$ and $\geq 70 \mathrm{yr}$ ).

Population Data $\mathrm{BC}$ does not include race or ethnicity, or genetic information such as $H L A-B^{\star} 5801$. The $H L A-B^{*} 5801$ allele is an important predictor of allopurinol-associated severe cutaneous adverse reactions; according to a meta-analysis, the risk of allopurinol-associated severe cutaneous adverse reactions among carriers of the allele was 97 times higher than among those who did not carry the allele. ${ }^{16}$ The allele is more prevalent among Asian and black patients than among white or Hispanic patients, thus explaining the racial and ethnic differences in the risk of allopurinol-associated severe cutaneous adverse reactions. ${ }^{15,17-22}$ Therefore, given the strong association between the
$H L A-B^{\star} 5801$ allele and the risk of allopurinol-associated severe cutaneous adverse reactions, and the high prevalence of $H L A-B^{\star} 5801$ among Asian populations, the 16 health regions within $B C$ were grouped into 4 region groups (Appendix 1, available at www.cmaj.ca/lookup/suppl/doi:10.1503/cmaj.190339/-/ DC1) based on the prevalence of Asian populations according to the 2016 Canadian Census data. ${ }^{23}$ This was then used as a surrogate for Asian race, which has the highest prevalence of $H L A-B^{\star} 5801 .{ }^{17,20,24,25}$ Secondarily, we analyzed regional prevalence of Asian populations as a continuous variable. Although black people also have an increased risk of allopurinol-associated severe cutaneous adverse reactions, ${ }^{8,11}$ with a high prevalence of $H L A-B^{*} 5801,{ }^{25}$ they constitute only $1.3 \%$ of the population of $\mathrm{BC}$ (v. $28.8 \%$ for Asian people). ${ }^{23}$ However, to assess the specificity of our surrogate measure, we similarly analyzed the regional prevalence of North American Aboriginal populations, the third largest racial or ethnic group (6.6\%) in BC after white (82.9\%) and Asian (28.8\%) populations.

\section{Statistical analysis}

We followed the study cohort members from the initiation of allopurinol until 1) hospital admission for allopurinol-associated severe cutaneous adverse reaction, 2) end of study period (Mar. 31, 2015), 3) end of the first 3 months of allopurinol exposure or 4) death, whichever came first. We calculated the overall risk of hospital admission for allopurinol-associated severe cutaneous adverse reactions per 1000 allopurinol initiators and the corresponding 95\% confidence interval $(\mathrm{Cl})$. We estimated the risk of hospital admission for allopurinol-associated severe cutaneous adverse reactions per 1000 allopurinol initiators according to history of heart disease as well as the aforementioned known and purported risk factors. We then calculated the relative risk (RR) associated with heart disease in Poisson regression models (with sandwich robust estimators), adjusting for demographic factors alone and additionally for known and purported risk factors for allopurinol-associated severe cutaneous adverse reactions. We confirmed the absence of collinearity among variables using the variance inflation factor.

We further examined the joint effects of combined demographic and clinical risk factors, including heart disease, on the risk of hospital admission for allopurinol-associated severe cutaneous adverse reactions following the approach taken by Keller and colleagues in their analysis of the US Medicaid population. ${ }^{8}$ We first investigated joint effects of heart disease and clinical covariates, chronic kidney disease and initial allopurinol dosage, as chronic kidney disease and heart disease often coexist $^{26,27}$ and allopurinol dosage is the key modifiable factor in patients with chronic kidney disease to reduce the risk of allopurinol-associated severe cutaneous adverse reactions. ${ }^{8,9,28}$ We then repeated the analysis with heart disease and demographic covariates. Region was categorized as a binary variable, with region groups 1 and 2 constituting high-risk regions with large Asian populations and region groups 3 and 4 constituting low-risk regions (Appendix 1). We also categorized age in the joint effects analysis as a binary variable, using age 70 as the cut-off point. 


\section{Ethics approval}

This study was exempted by the Partners Human Research Committee.

\section{Results}

\section{Cohort characteristics}

We identified 130325 allopurinol initiators in the database. The characteristics of patients with and without heart disease are shown in Table 1. Patients with heart disease tended to be older and had higher prevalence of chronic kidney disease, diabetes and diuretic use.

\section{Incidence, timing of hospital admission and mortality} Among the 130325 allopurinol initiators, we identified 109 cases of hospital admission for allopurinol-associated severe cutaneous adverse reactions occurring within 3 months of allopurinol initiation (Table 2). The overall risk of hospital admission for allopurinol-associated severe cutaneous adverse reactions was 1 out of 1196 allopurinol initiators. The risk of hospital admission for allopurinol-associated severe cutaneous adverse reactions became apparent within 10 days of allopurinol initiation, peaked around 1 month after initiation and declined progressively thereafter until the end of the 3-month period (Appendix 2, available at www.cmaj.ca/lookup/suppl/doi:10.1503/cmaj.190339/-/DC1). Among the patients admitted to hospital for allopurinolassociated severe cutaneous adverse reactions, 13 died within 60 days of the hospital admission, yielding a mortality rate of $12 \%$.

\section{Risk of hospital admission by heart disease status}

Among patients with heart disease, 1 in 655 allopurinol initiators $(1.53,95 \% \mathrm{Cl} 1.10-2.06$, per 1000$)$ were admitted to the hospital for allopurinol-associated severe cutaneous adverse reactions, versus 1 in 1548 allopurinol initiators $(0.65,95 \% \mathrm{Cl} 0.50-0.82$, per $1000)$ among those without heart disease. In the age- and sexadjusted analysis, heart disease was associated with an $84 \%$ higher risk of hospital admission for allopurinol-associated severe cutaneous adverse reactions (RR 1.84, 95\% Cl 1.23-2.76). After adjustment for other covariates, the RR attenuated but remained significant (multivariable $\mathrm{RR} 1.55,95 \% \mathrm{Cl} 1.01-2.37$ ).

\begin{tabular}{|c|c|c|c|}
\hline \multirow[b]{2}{*}{ Characteristic } & \multicolumn{2}{|c|}{ No. $(\%)$ of patients* } & \multirow{2}{*}{$\begin{array}{c}\text { Standardized } \\
\text { mean } \\
\text { difference } †\end{array}$} \\
\hline & $\begin{array}{l}\text { Heart disease } \\
n=28176\end{array}$ & $\begin{array}{c}\text { No heart disease } \\
n=102149\end{array}$ & \\
\hline Age, yr & & & 0.80 \\
\hline Mean \pm SD & $70.2 \pm 11.6$ & $59.9 \pm 14.0$ & \\
\hline$<60$ & $5401(19.2)$ & $48494(47.5)$ & \\
\hline $60-70$ & $7301(25.9)$ & $27271(26.7)$ & \\
\hline$\geq 70$ & $15454(54.9)$ & $26384(25.8)$ & \\
\hline Sex, female & $9328(33.1)$ & $27870(27.3)$ & 0.13 \\
\hline Region $\ddagger$ & & & 0.21 \\
\hline Richmond & $1010(3.6)$ & $4574(4.5)$ & \\
\hline Vancouver and Fraser North & $9414(33.4)$ & $24994(24.5)$ & \\
\hline North Shore and Fraser South & $5687(20.2)$ & $20998(20.6)$ & \\
\hline Remainder & $12065(42.8)$ & $51583(50.5)$ & \\
\hline \multicolumn{4}{|l|}{ Comorbidities } \\
\hline Ischemic heart disease & $24537(87.1)$ & 0 & 3.67 \\
\hline Heart failure & $10076(35.8)$ & 0 & 1.06 \\
\hline Chronic kidney disease & $7138(25.3)$ & $8736(8.6)$ & 0.46 \\
\hline Diabetes & $12111(43.0)$ & $20759(20.3)$ & 0.50 \\
\hline Gout & $18104(64.3)$ & $61660(60.4)$ & 0.08 \\
\hline \multicolumn{4}{|l|}{ Medications } \\
\hline Diuretics & $16415(58.3)$ & $26124(25.6)$ & 0.70 \\
\hline Initial allopurinol dosage $>100 \mathrm{mg} / \mathrm{d}$ & $16333(58.0)$ & $66520(65.1)$ & 0.15 \\
\hline \multicolumn{4}{|c|}{$\begin{array}{l}\text { Note: } \mathrm{SD}=\text { standard deviation. } \\
\text { *Unless stated otherwise. } \\
\text { † Standardized mean differences }>0.1 \text { are considered meaningful. } \\
\text { †Regions are grouped by proportion of Asian people in the population according to data from the } 2016 \text { Canadian } \\
\text { Census (Appendix } 1 \text {, available at www.cmaj.ca/lookup/suppl/doi:10.1503/cmaj. } 190339 /-/ D C 1 \text { ) and are used here as a } \\
\text { surrogate for Asian race, which has the highest prevalence of the HLA-B*5801 polymorphism. }\end{array}$} \\
\hline
\end{tabular}


Table 2: Risk of hospital admission for allopurinol-associated severe cutaneous adverse reactions, by purported risk factor

\begin{tabular}{|c|c|c|c|c|c|c|}
\hline \multirow[b]{2}{*}{ Risk factor } & \multicolumn{2}{|c|}{ No. $(\%)$} & \multirow{2}{*}{$\begin{array}{l}\text { Risk of hospital } \\
\text { admission for } \\
\text { allopurinol-associated } \\
\text { severe cutaneous } \\
\text { adverse reactions per } \\
1000 \text { patients }(95 \% \mathrm{Cl})\end{array}$} & \multicolumn{3}{|c|}{ Relative risk $(95 \% \mathrm{Cl})$} \\
\hline & $\begin{array}{l}\text { Allopurinol } \\
\text { initiators }\end{array}$ & $\begin{array}{c}\text { Hospital } \\
\text { admissions for } \\
\text { severe cutaneous } \\
\text { adverse reactions }\end{array}$ & & $\begin{array}{l}\text { Age- and } \\
\text { sex-adjusted }\end{array}$ & $\begin{array}{c}\text { Primary } \\
\text { multivariable }^{*}\end{array}$ & $\begin{array}{c}\text { Secondary } \\
\text { multivariable }\end{array}$ \\
\hline All & $130325(100)$ & 109 & $0.84(0.69-1.01)$ & - & - & - \\
\hline \multicolumn{7}{|l|}{ Sex } \\
\hline Male & $93127(71.5)$ & $49(45.0)$ & $0.53(0.39-0.70)$ & 1.0 & 1.0 & 1.0 \\
\hline Female & $37198(28.5)$ & $60(55.0)$ & $1.61(1.23-2.08)$ & $2.45(1.67-3.61)$ & $2.48(1.67-3.70)$ & $2.45(1.65-3.65)$ \\
\hline \multicolumn{7}{|l|}{ Age, yr } \\
\hline Continuous, 1-year age increase & - & - & - & - & $1.02(1.00-1.04)$ & - \\
\hline$<60$ & $53895(41.4)$ & $23(21.1)$ & $0.43(0.27-0.64)$ & 1.0 & - & 1.0 \\
\hline $60-70$ & $34572(26.5)$ & $21(19.3)$ & $0.61(0.38-0.93)$ & $1.76(1.37-2.25)$ & - & $2.24(1.33-3.78)$ \\
\hline$\geq 70$ & $41858(32.1)$ & $65(59.6)$ & $1.55(1.20-1.98)$ & $3.09(1.89-5.06)$ & - & $5.04(1.77-14.30)$ \\
\hline \multicolumn{7}{|l|}{ Region $\ddagger$} \\
\hline Continuous, per $1 \%$ Asian increase & - & - & - & - & $1.03(1.02-1.04)$ & - \\
\hline Richmond & $5584(4.3)$ & $8(7.3)$ & $1.43(0.62-2.82)$ & $4.66(2.66-8.15)$ & - & $4.67(2.65-8.25)$ \\
\hline Vancouver and Fraser North & $34408(26.4)$ & $54(49.5)$ & $1.57(1.18-2.05)$ & $2.79(1.92-4.05)$ & - & $2.80(1.92-4.08)$ \\
\hline North Shore and Fraser South & $26685(20.5)$ & $15(13.8)$ & $0.56(0.31-0.93)$ & $1.67(1.39-2.01)$ & - & $1.67(1.38-2.02)$ \\
\hline Remainder & $63648(48.8)$ & $32(29.4)$ & $0.50(0.34-0.71)$ & 1.0 & - & 1.0 \\
\hline \multicolumn{7}{|l|}{ Heart disease } \\
\hline Yes & $28176(21.6)$ & $43(39.4)$ & $1.53(1.10-2.06)$ & $1.84(1.23-2.76)$ & $1.60(1.04-2.44)$ & $1.55(1.01-2.37)$ \\
\hline No & $102149(78.4)$ & $66(60.6)$ & $0.65(0.50-0.82)$ & 1.0 & 1.0 & 1.0 \\
\hline \multicolumn{7}{|l|}{ Chronic kidney disease } \\
\hline Yes & 15874 (12.2) & $26(23.9)$ & $1.64(1.07-2.40)$ & $1.70(1.08-2.67)$ & $1.86(1.16-2.99)$ & $1.88(1.17-3.02)$ \\
\hline No & $114451(87.8)$ & $83(76.1)$ & $0.73(0.58-0.90)$ & 1.0 & 1.0 & 1.0 \\
\hline \multicolumn{7}{|l|}{ Diabetes } \\
\hline Yes & $32870(25.2)$ & $36(33.0)$ & $1.10(0.77-1.52)$ & $1.12(0.75-1.68)$ & $0.96(0.63-1.46)$ & $0.95(0.62-1.45)$ \\
\hline No & 97455 (74.8) & $73(67.0)$ & $0.75(0.59-0.94)$ & 1.0 & 1.0 & 1.0 \\
\hline \multicolumn{7}{|l|}{ Gout } \\
\hline Yes & 79764 (61.2) & $57(52.3)$ & $0.71(0.54-0.93)$ & $0.80(0.55-1.16)$ & $0.80(0.54-1.16)$ & $0.80(0.55-1.17)$ \\
\hline No & $50561(38.8)$ & $52(47.7)$ & $1.03(0.77-1.35)$ & 1.0 & 1.0 & 1.0 \\
\hline \multicolumn{7}{|l|}{ Diuretic use } \\
\hline Yes & 42539 (32.6) & 55 (50.5) & $1.29(0.97-1.68)$ & $1.32(0.88-1.98)$ & $1.27(0.84-1.94)$ & $1.26(0.83-1.92)$ \\
\hline No & 87786 (67.4) & $54(49.5)$ & $0.62(0.46-0.80)$ & 1.0 & 1.0 & 1.0 \\
\hline \multicolumn{7}{|l|}{ Initial allopurinol dosage } \\
\hline$>100 \mathrm{mg} / \mathrm{d}$ & $82853(63.6)$ & $86(78.9)$ & $1.04(0.83-1.28)$ & $2.53(1.59-4.02)$ & $2.79(1.75-4.45)$ & $2.78(1.75-4.43)$ \\
\hline$\leq 100 \mathrm{mg} / \mathrm{d}$ & $47472(36.4)$ & $23(21.1)$ & $0.48(0.31-0.73)$ & 1.0 & 1.0 & 1.0 \\
\hline $\begin{array}{l}\text { Note: } \mathrm{Cl}=\text { confidence interval } \\
\text { *Adjusted for sex, age (continuous), \% } \\
\text { †Adjusted for sex, age, region, heart dis } \\
\text { †Regions are grouped by proportion of } \\
\text { doi:10.1503/(cmaj.190339/-/DC1) and ar }\end{array}$ & $\begin{array}{l}n \text { (continuous) } \\
\text { e, chronic kidn } \\
\text { an people in th } \\
\text { sed here as a s }\end{array}$ & $\begin{array}{l}\text { disease, chronic ki } \\
\text { ase, diabetes, gou } \\
\text { lation according to } \\
\text { for Asian race, } \mathrm{wl}\end{array}$ & $\begin{array}{l}\text { sease, diabetes, gout, } \\
\text { ic use and initial allopu } \\
\text { om the } 2016 \text { Canadian } \\
\text { the highest prevalence }\end{array}$ & $\begin{array}{l}\text { use and initia } \\
\text { losage. } \\
\text { s Appendix } 1 \text {, } \\
H L A-B^{\star} 5801 \mathrm{pc}\end{array}$ & inol dosage. & uppl/ \\
\hline
\end{tabular}

Risk of hospital admission by demographic and clinical risk factors

Female sex (multivariable RR 2.45, 95\% Cl 1.65-3.65), older age (age 60-70 yr: multivariable RR 2.24, 95\% Cl 1.33-3.78; age $\geq 70$ yr: multivariable RR 5.04, 95\% Cl 1.77-14.30) and residence in regions with a higher proportion of people of Asian ethnicity
(Richmond: multivariable RR 4.67, 95\% Cl 2.65-8.25; Vancouver and Fraser North: multivariable RR 2.80, 95\% Cl 1.92-4.08; North Shore and Fraser South: multivariable RR 1.67, 95\% Cl 1.38-2.02) were the demographic variables independently associated with risk of hospital admission for allopurinol-associated severe cutaneous adverse reactions in the multivariable analysis (Table 2). 
We also analyzed age as a continuous variable and found that each 1-year increase in age was associated with a multivariable RR of 1.02 (95\% Cl 1.00-1.04), with other associations remaining similar. We considered prevalence of Asian population within a health region as a continuous variable and found that each $1 \%$ increase in Asian prevalence was associated with a multivariable RR of 1.03 (95\% Cl 1.02-1.04). We also considered the prevalence of North American Aboriginal populations and found a null association (RR 0.97, 95\% Cl 0.91-1.04), without affecting the remainder of results.

Chronic kidney disease (multivariable RR 1.88, 95\% Cl 1.173.02) and initial allopurinol dosage greater than $100 \mathrm{mg} / \mathrm{d}$ (multivariable $\mathrm{RR} 2.78,95 \% \mathrm{Cl} 1.75-4.43$ ) were the clinical variables independently associated with risk of hospital admission for allopurinol-associated severe cutaneous adverse reactions in the multivariable analysis. Gout and diabetes were not associated with increased risk of hospital admission for these reactions. Additionally, diuretic use, a previously suspected risk factor, was not associated with an increased risk of hospital admission for allopurinol-associated severe cutaneous adverse reactions (multivariable RR 1.26, 95\% Cl 0.83-1.92) (Table 2). Overall event rates were low, even among those with chronic kidney disease, who had the highest absolute event rate observed (1.64 per 1000 patients).

We tested the interactions terms for heart disease and each of the other risk factors for allopurinol-associated severe cutaneous adverse reactions and found no significant interactions, with $p$ values ranging from 0.1 to 0.8 .

\section{Risk of hospital admission by heart disease combined with chronic kidney disease and initial allopurinol dosage}

Patients with heart disease and chronic kidney disease who were started on allopurinol at a dosage of greater than $100 \mathrm{mg} / \mathrm{d}$ had an 11-fold higher risk of hospital admission for allopurinolassociated severe cutaneous adverse reactions than patients without heart disease or chronic kidney disease who were started at a dosage of $100 \mathrm{mg} / \mathrm{d}$ or less (multivariable RR 11.13, 95\% Cl 4.66-26.58) (Table 3). Among high-risk patients with both heart disease and chronic kidney disease, a lower initial allopurinol dosage of $100 \mathrm{mg} / \mathrm{d}$ or less reduced the multivariable adjusted RR from 11.13 to 2.28 (95\% Cl 0.68-7.58). Even among patients without chronic kidney disease, patients with heart disease who were started on allopurinol at a dosage of greater than $100 \mathrm{mg} / \mathrm{d}$ had a threefold increased risk of hospital admission for allopurinol-associated severe cutaneous adverse reactions (multivariable RR 3.44, 95\% Cl 1.52-7.81).

\section{Risk of hospital admission by heart disease combined with demographic factors}

Older women ( $\geq 70 \mathrm{yr}$ ) with heart disease living in a high-risk region (i.e., region with a large Asian population) had a 23-fold higher risk of hospital admission for allopurinol-associated severe cutaneous adverse reactions than younger men $(<70 \mathrm{yr}$ ) without heart disease living in a low-risk region (multivariable RR 22.61, 95\% Cl 7.57-67.60) (Table 4). Presence of comorbid heart disease was consistently associated with higher multivariable adjusted RRs than no heart disease across all combinations of demographic factors, with the exception of 2 categories (women aged $<70 \mathrm{yr}$ living in a high-risk region and men aged $\geq 70 \mathrm{yr}$ living in a high-risk region), where the sample sizes for the cases were low.

\section{Interpretation}

In this general population-based study, heart disease was independently associated with an increased risk of hospital admission for allopurinol-associated severe cutaneous adverse reactions. Furthermore, heart disease in combination with other risk factors significantly augmented the risk of hospital admission for these reactions. For example, patients with heart disease and chronic kidney disease who were started on allopurinol at an initial dosage of greater than $100 \mathrm{mg} / \mathrm{d}$ had an 11-fold increased risk of hospital admission for allopurinol-associated severe cutaneous adverse reactions compared with patients without heart disease or chronic kidney disease who were started on allopurinol at an initial dosage of $100 \mathrm{mg} / \mathrm{d}$ or less. Additionally, when allopurinol is prescribed to patients with cardiorenal

Table 3: Heart disease and risk of hospital admission for allopurinol-associated severe cutaneous adverse reactions, by presence of chronic kidney disease and initial allopurinol dosage

(+) Heart disease

\begin{tabular}{|c|c|c|}
\hline Variable & $\begin{array}{c}\text { Initial } \\
\text { allopurinol } \\
\text { dosage }\end{array}$ & $\begin{array}{l}\text { Risk of hospital admission } \\
\text { for allopurinol-associated } \\
\text { severe cutaneous adverse } \\
\text { reactions per } 1000 \text { patients } \\
(95 \% \mathrm{Cl})\end{array}$ \\
\hline \multirow{2}{*}{$\begin{array}{l}\text { (+) Chronic } \\
\text { kidney disease }\end{array}$} & $>100 \mathrm{mg} / \mathrm{d}$ & $4.45(2.44-7.47)$ \\
\hline & $\leq 100 \mathrm{mg} / \mathrm{d}$ & $1.00(0.27-2.56)$ \\
\hline \multirow{2}{*}{$\begin{array}{l}\text { (-) Chronic } \\
\text { kidney disease }\end{array}$} & $>100 \mathrm{mg} / \mathrm{d}$ & $1.29(0.75-2.06)$ \\
\hline & $\leq 100 \mathrm{mg} / \mathrm{d}$ & $1.02(0.44-2.01)$ \\
\hline
\end{tabular}

(-) Heart disease

Risk of hospital admission for allopurinol-associated

Multivariable

relative risk* $(95 \% \mathrm{Cl})$

$11.13(4.66-26.58)$

$2.28(0.68-7.58)$

$3.44(1.52-7.81)$

$2.41(0.92-6.64)$ reactions per 1000 patients severe cutaneous adverse $(95 \% \mathrm{Cl})$

\section{Multivariable relative risk ${ }^{\star}$ $(95 \% \mathrm{Cl})$}
$1.41(0.52-3.06)$
$0.45(0.05-1.61)$
$0.79(0.58-1.04)$
$0.29(0.13-0.55)$

$4.52(1.60-12.78)$

$1.24(0.27-5.80)$

$3.06(1.50-6.23)$

1.00 (Ref.)

Note: $\mathrm{Cl}$ = confidence interval, Ref. = reference category

*Adjusted for age, sex, region, diabetes, diuretics and gout. 
Table 4: Heart disease and risk of hospital admission for allopurinol-associated severe cutaneous adverse reactions, by

demographic profiles

(+) Heart disease

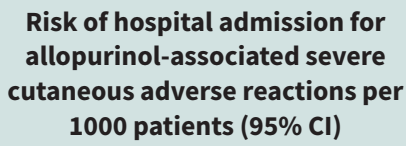

Variable

Female sex, region with high

Asian populationt; age, $\mathrm{yr}$

$\geq 70$

$3.65(1.57-7.19)$

$1.44(0.17-5.21)$

$22.61(7.57-67.60)$

$9.13(1.82-45.83)$

Multivariable

relative risk *

$(95 \% \mathrm{Cl})$

$<70$

Female sex, region with low

Asian populationt; age, yr

$\begin{array}{ll}\geq 70 & 2.30(1.05-4.37) \\ <70 & 1.63(0.34-4.78)\end{array}$

13.76 (4.67-40.55)

$9.68(2.35-39.80)$

$0.99(0.43-1.95)$

$0.67(0.29-1.32)$

$6.42(2.18-18.87)$

$4.38(1.51-12.73)$

Male sex, region with high

Asian populationt; age, $\mathrm{yr}$

$\geq 70$

$2.72(1.17-5.36)$

$17.10(5.78-50.55)$

$1.28(0.42-2.99)$

$8.43(2.56-27.77)$

$2.77(1.38-4.96)$

$0.34(0.12-0.73)$

$18.28(6.73-49.68)$

$<70$

Male sex, region with low

Asian populationt; age, $\mathrm{yr}$

$\begin{array}{ll}\geq 70 & 0.93(0.34-2.03) \\ <70 & 0.36(0.04-1.30)\end{array}$

$5.48(1.70-17.56)$

$2.27(0.45-11.34)$
(-) Heart disease
Risk of hospital admission for allopurinol-associated severe cutaneous adverse reactions per 1000 patients $(95 \% \mathrm{Cl})$
Multivariable

relative risk ${ }^{\star}$

$(95 \% \mathrm{Cl})$

Note: $\mathrm{Cl}=$ confidence interval, Ref. = reference category.

*Adjusted for chronic kidney disease, initial allopurinol dosage, diabetes, diuretics and gout.

tProportion of Asian people in the population by region, according to data from the 2016 Canadian Census, is used here as a surrogate for Asian race, which has the highest prevalence of the HLA-B*5801 polymorphism.

comorbidities, a starting dosage of $100 \mathrm{mg} / \mathrm{d}$ or less, as recommended by a guideline from the American College of Rheumatology, ${ }^{29}$ may reduce the risk of hospital admission for allopurinolassociated severe cutaneous adverse reactions by nearly fivefold (from 11.13 to 2.28 ).

Our findings are applicable to patients with gout, in whom there is a high prevalence of comorbid heart disease ${ }^{30-33}$ and chronic kidney disease. ${ }^{34-36}$ In light of the US Food and Drug Administration's warning ${ }^{2}$ regarding potential cardiovascular adverse effects related to febuxostat, ${ }^{37}$ allopurinol use among patients receiving treatment for gout who also have chronic kidney disease and heart disease may be set to increase. These findings may also have implications for clinical trials of allopurinol among at-risk populations. For example, in the Preventing Early Renal Loss in Diabetes (PERL) trial involving patients with type 1 diabetes and chronic kidney disease, ${ }^{38}$ all participants were screened for $H L A-B{ }^{\star} 5801$ as a safety measure given the increased risk of allopurinol-associated severe cutaneous adverse reactions in patients with chronic kidney disease. In contrast, the ALLHEART (Allopurinol and Cardiovascular Outcomes in Patients with Ischaemic Heart Disease) study, which aims to enroll thousands of patients with ischemic heart disease, including patients with chronic kidney disease, implemented no safety measures against allopurinol-associated severe cutaneous adverse reaction and rapidly escalated the allopurinol dosage in the study protocol. ${ }^{39}$
Our findings suggest that heart disease, like chronic kidney disease, is a risk factor for allopurinol-associated severe cutaneous adverse reactions that warrants adoption of precautionary measures against these reactions, such as low-dosage allopurinol initiation or screening for $H L A-B^{\star} 5801$.

The mechanisms behind the independent association between heart disease and hospital admission for allopurinolassociated severe cutaneous adverse reactions remain unclear. Stevens-Johnson syndrome and toxic epidermal necrolysis are considered to be a delayed-type hypersensitivity reaction mediated by genetic predisposition (especially human leukocyte antigen alleles), drug metabolism, and T- and NK-cell mediated cytotoxicity. ${ }^{40,41}$ Future research could clarify whether any of the genetic predispositions or immunologic milieu implicated in the development of Stevens-Johnson syndrome and toxic epidermal necrolysis may also be involved in heart disease pathogenesis. There are also a few case reports of a potential interaction between allopurinol and angiotensin-converting enzyme inhibitors; however, only one such case involved a cutaneous reaction. ${ }^{42-44}$

Our study also confirmed previously reported demographic and clinical risk factors associated with hospital admission for allopurinol-associated severe cutaneous adverse reactions using a Canadian general population database. Older age, female sex, presence of chronic kidney disease, and initial allopurinol dosage 
of greater than $100 \mathrm{mg} / \mathrm{d}$ were all independently associated with hospital admission for allopurinol-associated severe cutaneous adverse reactions, consistent with those found in the Taiwanese $^{6}$ and US Medicaid ${ }^{8}$ populations. Furthermore, our study did not find that diuretics were associated with increased risk of allopurinol-associated severe cutaneous adverse reactions, in contrast to prior studies which suggested a link. ${ }^{45}$

Although information on race was not available through Population Data BC, we used prevalence of Asian residents in a health region as a surrogate marker of at-risk race and observed results consistent with those obtained from the US Medicaid population. ${ }^{8}$ We also evaluated and found a null association between North American Aboriginal populations and the risk of allopurinol-associated severe cutaneous adverse reactions. Overall low prevalence, lack of sufficient variation across the regions, and lack of association with this ethnicity group could all potentially explain this null association. To this end, while we do not know the specific prevalence of $H L A-B{ }^{*} 5801$ in North American Aboriginal populations in British Columbia, data on US Native Americans suggest that its frequency is similar to that in white populations. Similarly, the other racial and ethnic groups in $\mathrm{BC}$ are too small $(<2 \%)$ for meaningful analyses.

We used a comprehensive province-wide database from BC, which has universal health care and thus is expected to capture nearly all health care use among its residents. Thus, this study is more generalizable than the US Medicaid study, which represents more underserved populations. By limiting the time period of allopurinol-associated severe cutaneous adverse reaction development to within 3 months of the first allopurinol prescription, we minimized any potential issues related to early drug discontinuation.

\section{Limitations}

As this study relied on ICD codes, misclassification of allopurinolassociated severe cutaneous adverse reactions and relevant comorbidities is possible. Although a prior study found a high degree of accuracy of the ICD codes for these reactions based on dermatologists' review of the medical records, ${ }^{6}$ validation of these codes has not been done in the BC database. However, any misclassification is expected to be nondifferential and thus bias the results toward the null, yielding conservative estimates of risk.

Another limitation of this study is the absence of objective data on patients' race. However, we incorporated census-derived prevalence of Asian populations as a surrogate marker of high-risk race and ethnicity reflecting underlying prevalence of $H L A-B^{\star} 5801,{ }^{25}$ and the results are closely consistent with what has been shown in other studies that incorporated either $H L A-B{ }^{\star} 5801$ or data on race and ethnicity. . $^{811,17,24}$

\section{Conclusion}

We found that heart disease was independently associated with an increased risk of hospital admission for allopurinol-associated severe cutaneous adverse reactions. Additionally, combinations of heart disease and other risk factors significantly augment the risk of hospital admission for these reactions. However, allopurinol- associated severe cutaneous adverse reactions are notably rare, even among those with multiple risk factors (e.g., 4.45 per 1000 people for those with chronic kidney disease, heart disease and initial allopurinol dosage $>100 \mathrm{mg} / \mathrm{d}$ ). We were also able to replicate previously reported associations between older age, female sex, chronic kidney disease, and higher initial allopurinol dosage on the risk of hospital admission for allopurinol-associated severe cutaneous adverse reactions in another general population-based database. Physicians who prescribe allopurinol should look for these risk factors so that they may consider initiating lowerdosage allopurinol and other precautions, which may prevent this rare but serious adverse reaction.

\section{References}

1. Kuo CF, Grainge MJ, Mallen C, et al. Rising burden of gout in the UK but continuing suboptimal management: a nationwide population study. Ann Rheum Dis 2015;74:661-7.

2. FDA adds Boxed Warning for increased risk of death with gout medicine Uloric (febuxostat) [safety announcement]. Silver Spring (MD): US Food and Drug Administration; 2019. Available: www.fda.gov/drugs/drug-safety-and-availability/ fda-adds-boxed-warning-increased-risk-death-gout-medicine-uloric-febuxostat (accessed 2019 Mar. 2).

3. ClinicalTrials.gov. 44 Studies found for: allopurinol cardiovascular. Available: https://clinicaltrials.gov/ct2/results?cond=\&term=allopurinol+cardiovascular\& cntry $=\&$ state $=\&$ city $=\&$ dist $=($ accessed 2019 Feb. 21) .

4. Rentoukas E, Tsarouhas K, Tsitsimpikou C, et al. The prognostic impact of allopurinol in patients with acute myocardial infarction undergoing primary percutaneous coronary intervention. Int J Cardiol 2010;145:257-8.

5. Noman A, Ang DS, Ogston S, et al. Effect of high-dose allopurinol on exercise in patients with chronic stable angina: a randomised, placebo controlled crossover trial. Lancet 2010;375:2161-7.

6. Yang $\mathrm{CY}$, Chen $\mathrm{CH}$, Deng ST, et al. Allopurinol use and risk of fatal hypersensitivity reactions: a nationwide population-based study in Taiwan. JAMA Intern Med 2015;175:1550-7.

7. Ng CY, Yeh YT, Wang CW, et al.; Taiwan Severe Cutaneous Adverse Reaction Consortium. Impact of the HLA-B( $\left.{ }^{\star}\right) 58: 01$ allele and renal impairment on allopurinolinduced cutaneous adverse reactions. J Invest Dermatol 2016;136:1373-81.

8. Keller SF, Lu N, Blumenthal KG, et al. Racial/ethnic variation and risk factors for allopurinol-associated severe cutaneous adverse reactions: a cohort study. Ann Rheum Dis 2018;77:1187-93.

9. Stamp LK, Taylor WJ, Jones PB, et al. Starting dose is a risk factor for allopurinol hypersensitivity syndrome: a proposed safe starting dose of allopurinol. Arthritis Rheum 2012;64:2529-36.

10. Saksit N, Tassaneeyakul W, Nakkam N, et al. Risk factors of allopurinolinduced severe cutaneous adverse reactions in a Thai population. Pharmacogenet Genomics 2017;27:255-63.

11. Lu N, Rai SK, Terkeltaub R, et al. Racial disparities in the risk of Stevens-Johnson Syndrome and toxic epidermal necrolysis as urate-lowering drug adverse events in the United States. Semin Arthritis Rheum 2016;46:253-8.

12. Canadian Institute for Health Information. Discharge Abstracts Database (Hospital separations file). Vancouver: Population Data BC. Data Extract. $\mathrm{MOH}$; revised 2018 Nov. 15. Available: www.popdata.bc.ca/data/health/dad (accessed 2019 Jan. 15).

13. PharmaNet. Vancouver: Population Data BC; updated 2018 Nov. 20. Available: www.popdata.bc.ca/data/health/PharmaNet (accessed 2019 Jan. 15).

14. About PopData. Vancouver: Population Data BC; updated 2018 Aug. 23. Available: www.popdata.bc.ca/aboutus (accessed 2019 Jan. 15).

15. Hung $\mathrm{SI}$, Chung WH, Liou LB, et al. HLA-B ${ }^{\star} 5801$ allele as a genetic marker for severe cutaneous adverse reactions caused by allopurinol. Proc Natl Acad Sci U SA 2005;102:4134-9.

16. Somkrua R, Eickman EE, Saokaew S, et al. Association of HLA-B*5801 allele and allopurinol-induced Stevens Johnson syndrome and toxic epidermal necrolysis: a systematic review and meta-analysis. BMC Med Genet 2011;12:118.

17. Kang HR, Jee YK, Kim YS, et al.; Adverse Drug Reaction Research Group in Korea. Positive and negative associations of HLA class I alleles with allopurinol-induced SCARs in Koreans. Pharmacogenet Genomics 2011;21:303-7.

18. Jung JW, Song WJ, Kim YS, et al. HLA-B58 can help the clinical decision on starting allopurinol in patients with chronic renal insufficiency. Nephrol Dial Transplant 2011;26:3567-72. 
19. Kaniwa N, Saito Y, Aihara M, et al. JSAR research group. HLA-B locus in Japanese patients with anti-epileptics and allopurinol-related Stevens-Johnson syndrome and toxic epidermal necrolysis. Pharmacogenomics 2008;9:1617-22.

20. Tassaneeyakul W, Jantararoungtong $T$, Chen $P$, et al. Strong association between HLA-B 5801 and allopurinol-induced Stevens-Johnson syndrome and toxic epidermal necrolysis in a Thai population. Pharmacogenet Genomics 2009;19:704-9.

21. Lonjou C, Borot N, Sekula P, et al. RegiSCAR study group. A European study of HLA-B in Stevens-Johnson syndrome and toxic epidermal necrolysis related to five high-risk drugs. Pharmacogenet Genomics 2008;18:99-107.

22. Zineh I, Mummaneni P, Lyndly J, et al. Allopurinol pharmacogenetics: assessment of potential clinical usefulness. Pharmacogenomics 2011;12:1741-9.

23. Census profile. 2016 Census. British Columbia [Province] and Canada [Country] (table). Ottawa: Statistics Canada; modified 2019 June 17. Cat no 98-316-X2016001. Available: www12.statcan.gc.ca/census-recensement/2016/dp-pd/prof/ search-recherche/lst/results-resultats.cfm?Lang=E\&TABID=1\&G=1\&Geo1=PR\& Code1=59\&Geo2=PR\&Code2=01\&GEOCODE=59 (accessed 2019 Feb. 6).

24. Ko T-M, Tsai C-Y, Chen S-Y, et al.; Taiwan Allopurinol-SCAR Consortium. Use of HLA-B ${ }^{\star} 58: 01$ genotyping to prevent allopurinol induced severe cutaneous adverse reactions in Taiwan: national prospective cohort study. BMJ 2015;351: h4848.

25. HLA allele frequencies. Allele*Frequencies in Worldwide Populations [database]. Available: www.allelefrequencies.net/hla6006a.asp?hla_locus_type=Classical\&hla_

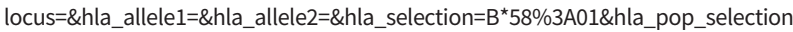

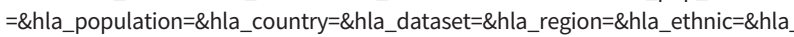
study=\&hla_order=order_1\&hla_sample_size_pattern=equal\&hla_sample_size= \&hla_sample_year_pattern=equal\&hla_sample_year=\&hla_level_pattern=equal\& hla_level=\&standard=a\&hla_show= (accessed 2019 Feb. 21).

26. Go AS, Chertow GM, Fan D, et al. Chronic kidney disease and the risks of death, cardiovascular events, and hospitalization. N Engl J Med 2004;351:1296-305.

27. Tuegel C, Bansal N. Heart failure in patients with kidney disease. Heart 2017; 103:1848-53.

28. Vargas-Santos AB, Neogi T. Management of gout and hyperuricemia in CKD. Am J Kidney Dis 2017;70:422-39.

29. Khanna D, Fitzgerald JD, Khanna PP, et al.; American College of Rheumatology. 2012 American College of Rheumatology guidelines for management of gout. Part 1: systematic nonpharmacologic and pharmacologic therapeutic approaches to hyperuricemia. Arthritis Care Res (Hoboken) 2012;64:1431-46.

30. Choi HK, Curhan G. Independent impact of gout on mortality and risk for coronary heart disease. Circulation 2007;116:894-900.
31. Abbott RD, Brand FN, Kannel WB, et al. Gout and coronary heart disease: the Framingham Study. J Clin Epidemiol 1988;41:237-42.

32. Krishnan E, Baker JF, Furst DE, et al. Gout and the risk of acute myocardial infarction. Arthritis Rheum 2006;54:2688-96.

33. Singh JA, Cleveland JD. Gout and the risk of myocardial infarction in older adults: a study of Medicare recipients. Arthritis Res Ther 2018;20:109.

34. Sattui SE, Singh JA, Gaffo AL. Comorbidities in patients with crystal diseases and hyperuricemia. Rheum Dis Clin North Am 2014;40:251-78.

35. Roughley M, Sultan AA, Clarson L, et al. Risk of chronic kidney disease in patients with gout and the impact of urate lowering therapy: a populationbased cohort study. Arthritis Res Ther 2018;20:243.

36. Kuo CF, Grainge MJ, Mallen C, et al. Comorbidities in patients with gout prior to and following diagnosis: case-control study. Ann Rheum Dis 2016;75:210-7.

37. White WB, Saag KG, Becker MA, et al. CARES Investigators. Cardiovascular safety of febuxostat or allopurinol in patients with gout. N Engl J Med 2018;378: $1200-10$.

38. A multicenter clinical trial of allopurinol to prevent kidney function loss in type 1 diabetes. ClinicalTrials.gov: NCT02017171. Available: https://clinicaltrials. gov/ct2/show/NCT02017171?term=PERL (accessed 2019 Jan. 15).

39. Mackenzie IS, Ford I, Walker A, et al.; ALL-HEART study group. Multicentre, prospective, randomised, open-label, blinded end point trial of the efficacy of allopurinol therapy in improving cardiovascular outcomes in patients with ischaemic heart disease: protocol of the ALL-HEART study. BMJ Open 2016;6: e013774.

40. Dodiuk-Gad RP, Chung WH, Valeyrie-Allanore L, et al. Stevens-Johnson syndrome and toxic epidermal necrolysis: an update. Am J Clin Dermatol 2015;16: 475-93.

41. Chung WH, Chang WC, Stocker SL, et al. Insights into the poor prognosis of allopurinol-induced severe cutaneous adverse reactions: the impact of renal insufficiency, high plasma levels of oxypurinol and granulysin. Ann Rheum Dis 2015;74:2157-64.

42. Ahmad S. Allopurinol and enalapril. Drug induced anaphylactic coronary spasm and acute myocardial infarction. Chest 1995;108:586.

43. Samanta A, Burden AC. Fever, myalgia, and arthralgia in a patient on captopril and allopurinol. Lancet 1984;1:679.

44. Pennell DJ, Nunan TO, O'Doherty MJ, et al. Fatal Stevens-Johnson syndrome in a patient on captopril and allopurinol. Lancet 1984;1:463.

45. Stamp LK, Day RO, Yun J. Allopurinol hypersensitivity: investigating the cause and minimizing the risk. Nat Rev Rheumatol 2016;12:235-42.
Competing interests: Hyon Choi reports a grant from the National Institutes of Health and research support from AstraZeneca, and consulting fees from Takeda, Selecta Biosciences and Horizon (all less than $\$ 10000)$. No other competing interests declared.

This article has been peer reviewed.

Affiliations: Division of Rheumatology, Allergy and Immunology (Yokose, McCormick, Choi), Massachusetts General Hospital, Boston, Mass.; Arthritis Research Canada (Lu, Xie, Li, Zheng, McCormick, Rai, Aviña-Zubieta, Choi), Richmond, BC; Faculty of Health Sciences (Xie), Simon Fraser University, Burnaby, BC; Division of Rheumatology (Aviña-Zubieta), University of British Columbia, Vancouver, BC
Contributors: All authors contributed to the conception and design of this work. Na Lu, Chio Yokose and Hyon Choi were responsible for the analysis, with all authors contributing to the interpretation of the data. Chio Yokose drafted the manuscript and all authors reviewed and revised the manuscript for important intellectual content. All authors gave final approval of the version to be published and agree to be accountable for all aspects of the work.

Funding: Chio Yokose is supported by the National Institutes of Health Ruth L. Kirschstein Institutional National Research Service Award (T32-AR-007258). Hui Xie receives partial support from NSERC (Natural Sciences and Engineering Research Council of Canada) Discovery RGPIN-2018-04313. Natalie McCormick is sup- ported by a fellowship award from the Canadian Institutes of Health Research (CIHR). Sharan Rai is supported by a Doctoral Foreign Study Award from CIHR. J. Antonio AviñaZubieta receives support as the BC Lupus Society Scholar and the Michael Smith Foundation Research Scholar. Hyon Choi is supported by the National Institutes of Health (AR060772). This study was funded by CIHR (Team Grant THC 135235).

Data sharing: Data are not available for sharing.

Accepted: Aug. 19, 2019

Correspondence to: Hyon Choi, hchoi@mgh. harvard.edu 